Wolfgang Scherf

\title{
Kommunaler Finanzausgleich
}

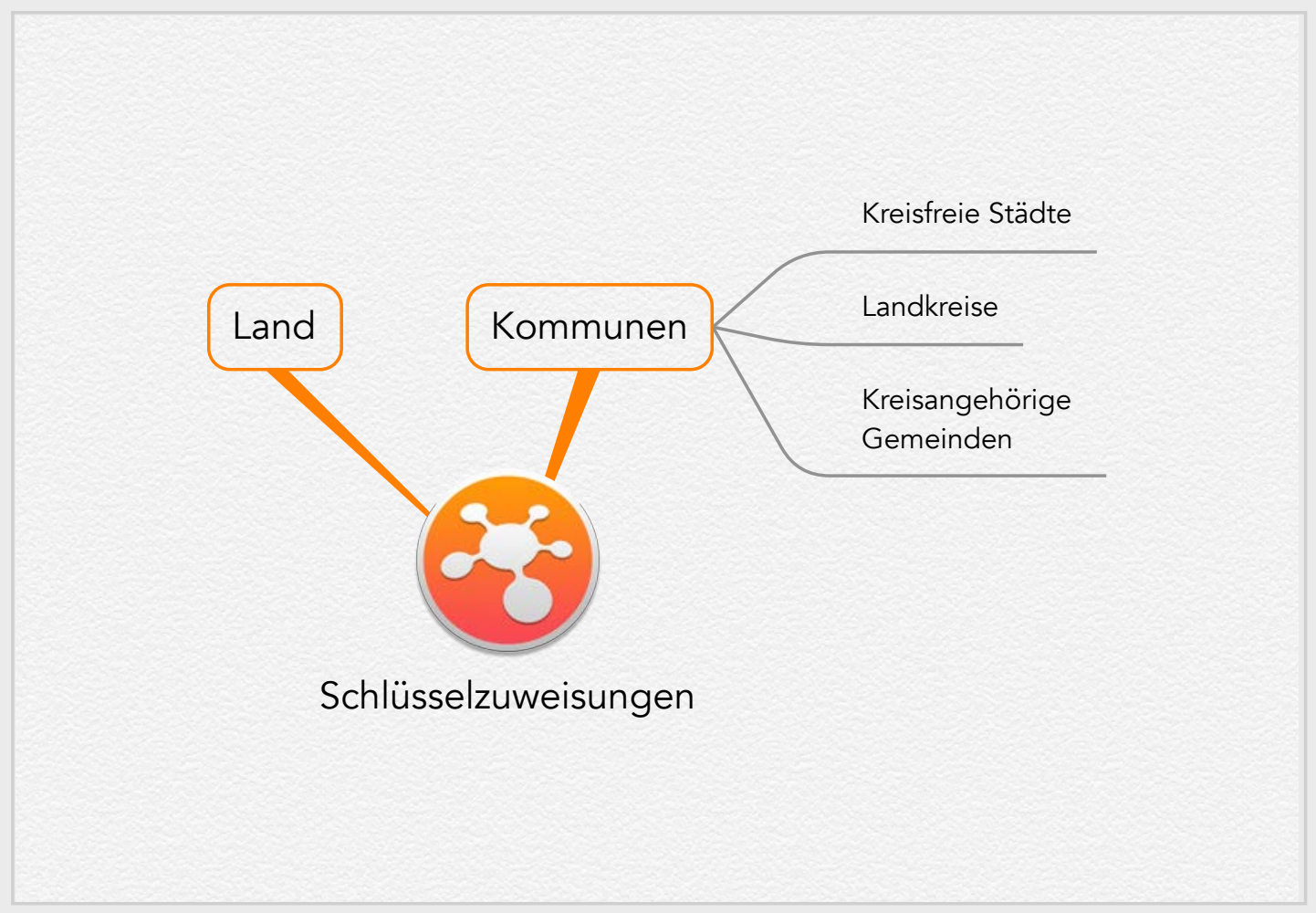

Prof. Dr. Wolfgang Scherf | Justus-Liebig-Universität Gießen | Licher Strasse 74 | 35394 Gießen 
Wolfgang Scherf

\section{Kommunaler Finanzausgleich}

Der kommunale Finanzausgleich (KFA) hat erhebliche Bedeutung für die Finanzierung der Gemeinden und Gemeindeverbände. Er dient der Aufstockung der Finanzkraft der kommunalen Ebene insgesamt sowie der Anpassung von Finanzkraft und Finanzbedarf bei den einzelnen kommunalen Gebietskörperschaften. Die Finanzausgleichssysteme variieren erheblich zwischen den deutschen Bundesländern, die für die konkreten Regelungen verantwortlich sind. Im Folgenden wird ein Überblick über zentrale Gestaltungsfragen und damit verbundene Probleme gegeben.

\section{Kommunaler Finanzausgleich im Überblick}

Im föderalen Staat werden staatliche Leistungen von unterschiedlichen Gebietskörperschaften erbracht. Die Finanzverfassung regelt die Verteilung der Aufgaben, Ausgaben und Einnahmen auf Bund, Länder und Gemeinden. Alle Gebietskörperschaften sollen ihre Aufgaben selbstverantwortlich wahrnehmen und benötigen hierfür eine angemessene Finanzautonomie. Unter dieser Voraussetzung können Dezentralisierung und föderaler Wettbewerb zu einer besseren Orientierung an den Bürgerpräferenzen und zur Steigerung der Effizienz im öffentlichen Sektor beitragen (vgl. Scherf 2014, S. 608 ff).

\subsection{Vertikaler Finanzausgleich}

Der Finanzausgleich im weiteren Sinne beginnt mit der vertikalen Verteilung der Aufgaben auf die verschiedenen Staatsebenen (vgl. zum KFA Scherf, 2013). Die Gemeinden erfüllen freiwillige und pflichtige Selbstverwaltungsaufgaben sowie Fremdverwaltungsaufgaben (vor allem Auftragsangelegenheiten). Aus den Aufgaben folgen die Ausgaben. Die größten Ausgabenblöcke sind die Personal- und Sozialausgaben. Die strukturelle Entwicklung der Ausgaben ist geprägt von einer Verdrängung der Investitionen durch die Sozialausgaben.

Hauptsächlich werden die Ausgaben aus Steuern und Finanzzuweisungen finanziert, die zusammen rund 75\% der kommunalen Einnahmen erbringen. Hinzu kommen Gebühren und Beiträge, Einnahmen aus wirtschaftlicher Tätigkeit, Konzessionsabgaben und Veräußerungserlöse. Die Gemeinden erhalten 15\% des Landesaufkommens der 
Lohnsteuer und veranlagten Einkommensteuer sowie 12\% der Abgeltungsteuer. Zudem sind sie mit 2,2\% am Aufkommen der Umsatzsteuer nach Abzug der Vorweganteile für den Bund beteiligt (effektiv ca. 2\%). Bei Gewerbesteuer und Grundsteuer verfügen die Gemeinden über ein Hebesatzrecht, das die kommunale Finanzautonomie stärkt.

Die Gemeinden sind zur Erfüllung ihrer Aufgaben auf Landeszuschüsse angewiesen. Daher müssen die Länder einen Teil ihrer Steuereinnahmen an die kommunale Ebene transferieren. Laut Grundgesetz sind die Bundesländer verpflichtet, ihre Gemeinden am Länderanteil an der Einkommensteuer, Körperschaftsteuer und Umsatzsteuer zu beteiligen (obligatorischer Steuerverbund). Zudem können die Länder die Einnahmen aus den Landessteuern oder aus dem Länderfinanzausgleich in den Steuerverbund einbeziehen (fakultativer Steuerverbund). Die Großzügigkeit eines Landes äußert sich freilich nicht in der Bereitschaft zum fakultativen Steuerverbund. Eine breitere Verbundmasse kann durch eine geringere Verbundquote leicht kompensiert werden.

\subsection{Horizontaler Finanzausgleich}

Neben der vertikalen Verteilung zwischen Land und Kommunen muss innerhalb der Gemeindeebene eine horizontale Aufteilung der Mittel stattfinden. Hierbei spielt die Zerlegung der Einkommensteuer eine zentrale Rolle. Sie richtet sich nach der Steuerleistung der Einwohner. Jedoch werden nur Einkommen bis zu Höchstbeträgen von 35.000 (Ledige) bzw. $70.000 €$ (Verheiratete) berücksichtigt. Dies vermindert die Streuung des Aufkommens zugunsten einkommensschwacher Gemeinden. Dennoch bestehen nach der Steuerverteilung erhebliche interkommunale Finanzkraftdifferenzen, die vor allem auf die Unterschiede in der Gewerbesteuerkraft zurückgehen.

Der kommunaler Finanzausgleich dient nicht nur der Erhöhung der kommunalen Gesamteinnahmen (fiskalisches Ziel), sondern vor allem der besseren Abstimmung von Finanzkraft und Finanzbedarf der kommunalen Gebietskörperschaften (distributives Ziel). Aus der Verbundmasse werden allgemeine und zweckgebundene Finanzzuweisungen gezahlt. Zweckzuweisungen dotieren bestimmte Aufgaben und Bedarfe. Der größte Teil entfällt auf Investitionszuweisungen. Daneben werden Kostenerstattungen für Auftragsangelegenheiten gewährt.

Allgemeine Finanzzuweisungen sind ungebundene Finanzmittel, die horizontale Ausgleichswirkungen erzielen sollen. Zum Abbau der Finanzkraftdifferenzen zwischen re- 
lativ armen und reichen Gemeinden tragen vor allem die Schlüsselzuweisungen bei. Das Verteilungssystem arbeitet nach folgendem Muster:

- Die Schlüsselmasse wird i.d.R. zunächst nach Quoten auf die Gruppen (1) kreisfreie Städte, (2) kreisangehörige Gemeinden und (3) Landkreise verteilt.

- Für Städte und Gemeinden wird eine Steuerkraftmesszahl und für die Landkreise eine Umlagekraftmesszahl ermittelt. Letztere basiert auf der Steuerkraft und den Schlüsselzuweisungen der kreisangehörigen Gemeinden.

- Die Steuerkraft resultiert aus den Anteilen an der Einkommen- und Umsatzsteuer sowie dem Grund- und Gewerbesteueraufkommen. Die Realsteuerkraft wird zu normierten Hebesätzen berechnet.

- Die Bedarfsmesszahl errechnet sich als Produkt aus Gesamtansatz und Grundbetrag. Der Gesamtansatz ist eine dimensionslose relative Bedarfsgröße. Der Grundbetrag sorgt für eine Anpassung der Ausgleichsansprüche an die Schlüsselmasse.

- Der Gesamtansatz besteht aus Haupt- und Ergänzungsansätzen. Der Hauptansatz fußt auf der Einwohnerzahl, die häufig durch eine Hauptansatzstaffel "veredelt" wird, um unterschiedliche Bedarfe und zentralörtliche Funktionen abzubilden.

- Die Ergänzungsansätze dienen der Berücksichtigung einer Vielzahl von Sonderbedarfen (z.B. Sozialhilfelasten, Schülerzahl) mit nicht immer klarer Berechtigung.

- Die allgemeinen Schlüsselzuweisungen kompensieren einen Teil der Differenz zwischen Steuerkraft- und Bedarfsmesszahl. Für besonders finanzschwache Gemeinden wird häufig ein Mindestprozentsatz an der Bedarfsmesszahl garantiert.

- Abundante Gemeinden, deren Steuerkraftmesszahl über ihrer Bedarfsmesszahl liegt, erhalten i.d.R. keine allgemeinen Schlüsselzuweisungen. Meistens wird ihre überdurchschnittliche Steuerkraft durch eine Finanzausgleichsumlage belastet.

\section{Grundmodell des kommunalen Finanzausgleichs}

Die skizzierten Elemente des kommunalen Finanzausgleichs haben in den einzelnen Bundesländern jeweils spezifische Ausprägungen und werden unterschiedlich kombiniert (vgl. Lenk, 2013). Im Folgenden geht es nicht um die Analyse der daraus resultierenden komplexen Systeme. Vielmehr werde ich ein elementares Ausgleichsystem entwerfen und dann einzelne prototypische Elemente realer Systeme hinzufügen, um de- 
ren Bedeutung zu prüfen (vgl. zur Modellierung von Ausgleichsystemen Kuhn, 1988, S. 15-58). Folgende Elemente charakterisieren das Grundmodell:

- Betrachtet werden nur Schlüsselzuweisungen, keine Zweckzuweisungen. Es gibt eine absolut fixierte Schlüsselmasse, aber keine Teilschlüsselmassen für Gebietskörperschaftsgruppen (kreisfreie Städte, Landkreise, kreisangehörige Gemeinden).

- Kreisfreie Städte wie kreisangehörige Gemeinden erhalten Schlüsselzuweisungen nach denselben Kriterien. Die Landkreise erhalten keine Schlüsselzuweisungen, sondern finanzieren sich allein über die Kreisumlage.

- Die Steuerkraft einer Gemeinde wird gemessen an ihren Gemeinschaftsteuereinnahmen zuzüglich der Realsteuern zu landesdurchschnittlichen Hebesätzen.

- Der Finanzbedarf einer Gemeinde wird gemessen an ihrer ungewichteten Einwohnerzahl multipliziert mit einem einheitlichen Grundbetrag. Damit wird ein gleichförmiger Pro-Kopf-Finanzbedarf unterstellt.

- $\quad$ Eine Differenz zwischen Bedarfs- und Steuerkraftmesszahl wird zu einem einheitlichen Auffüllungs- bzw. Abschöpfungssatz ausgeglichen.

Dieses Modell impliziert einen horizontalen Finanzkraftausgleich, der Unterschiede in der Pro-Kopf-Finanzkraft der Gemeinden linear nivelliert.

\subsection{Rein horizontaler Finanzausgleich}

Modelliert man das Verfahren als direkten horizontalen Ausgleich, so ergibt sich die jeweilige Pro-Kopf-Auffüllung bzw. Abschöpfung $(Z)$ aus der Differenz zwischen der durchschnittlichen Pro-Kopf-Steuerkraft $\left(\mathrm{S}_{\varnothing}\right)$ und der Pro-Kopf-Steuerkraft der Gemeinde (S) multipliziert mit dem Ausgleichsatz (a):

(1) $\quad Z=a\left(S_{\varnothing}-S\right)$

Dementsprechend beläuft sich die kommunale Pro-Kopf-Finanzkraft (F) nach Finanzausgleich auf:

(2) $\quad \mathrm{F}=\mathrm{S}+\mathrm{Z}=\mathrm{S}+\mathrm{a}\left(\mathrm{S}_{\varnothing}-\mathrm{S}\right)$

Der Ausgleichsatz zeigt die Nivellierungsintensität des Systems an. Relativ zum Durchschnitt werden alle Finanzkraftpositionen proportional herauf- bzw. herabgesetzt. Auffüllungen und Abschöpfungen decken sich in der Summe, so dass die Umverteilungs- 
komponenten sich aufheben. Daher entspricht die mittlere Finanzkraft der Gemeinden nach Finanzausgleich ihrer durchschnittlichen Steuerkraft:

(3) $\mathrm{Z}_{\varnothing}=0 \quad$ und $\quad \mathrm{F}_{\varnothing}=\mathrm{S}_{\varnothing}$

\subsection{Vertikaler Ausgleich mit horizontalem Effekt}

In der Praxis ist der kommunale Finanzausgleich nicht als direkter horizontaler Ausgleich gestaltet, sondern als vertikaler Ausgleich mit horizontalem Effekt. Das Land stockt die Finanzkraft der kommunalen Ebene aus seinen Mitteln auf und verteilt die Schlüsselmasse so auf die einzelnen Kommunen, dass die Finanzkraftdifferenzen verkleinert werden. Zur durchschnittlichen Pro-Kopf-Finanzkraft der Gemeinden nach Finanzausgleich rechnen neben der durchschnittlichen Steuerkraft nun auch die Schlüsselzuweisungen pro Kopf $\left(Z_{\varnothing}\right)$ als vertikale Ausgleichskomponente:

(4) $\quad F_{\varnothing}=S_{\varnothing}+Z_{\varnothing}$

Die Schlüsselzuweisungen orientieren sich an der Differenz zwischen der Steuerkraftmesszahl und einer Bedarfsmesszahl. Letztere ist im kommunalen Finanzausgleich keine absolut fixierte, sondern eine relative Größe, die den Bedarf einer Gebietskörperschaft im Vergleich zu anderen Gebietskörperschaften ausdrückt. Der durchschnittliche Bedarf pro Einwohner wird durch den flexiblen Grundbetrag $\left(\mathrm{B}_{\varnothing}\right)$ abgebildet. Der ProKopf-Bedarf einer Gemeinde (B) kann davon abweichen (vgl. Abschnitt 3.3). Über den Grundbetrag werden die Ausgleichsansprüche so gesteuert, dass die vorhandenen Ausgleichsmittel ausgeschöpft werden.

Bei horizontalem Ausgleich mit linearem Tarif heben sich die Umverteilungseffekte im Mittel auf und der Grundbetrag stimmt mit der durchschnittlichen Pro-Kopf-Finanzkraft überein $\left(B_{\varnothing}=F_{\varnothing}\right)$. Dies ist bei vertikalem Ausgleich mit horizontalem Effekt anders, weil die Schlüsselzuweisungen die Finanzkraft zugleich vergrößern und nivellieren.

Die Schlüsselzuweisungen einer Gemeinde richten sich nach der Differenz zwischen Bedarfs- und Steuerkraftmesszahl, wobei der Bedarf im einfachsten Fall durch den ungewichteten Grundbetrag gemessen wird:

(5) $\quad Z=a\left(B_{\varnothing}-S\right)$

Um die Schlüsselmasse auszuschöpfen, muss der Grundbetrag so festgelegt werden, dass eine durchschnittlich steuerstarke Gemeinde $\left(S=S_{\varnothing}\right)$ die mittlere Schlüsselzuwei- 
sung pro Einwohner erhält und damit auch nach Finanzausgleich über eine mittlere Finanzkraft verfügt:

(6) $\mathrm{Z}_{\varnothing}=\mathrm{a}\left(\mathrm{B}_{\varnothing}-\mathrm{S}_{\varnothing}\right) \quad \Rightarrow \quad \mathrm{B}_{\varnothing}=\mathrm{S}_{\varnothing}+\mathrm{Z}_{\varnothing} / \mathrm{a}$

Einsetzen des Grundbetrags, der das System zum Ausgleich bringt, in Gleichung (5) führt zu folgender Bestimmungsgleichung für die Schlüsselzuweisungen:

(7) $\quad Z=Z_{\varnothing}+a\left(S_{\varnothing}-S\right)$

Sie zerlegt den kommunalen Finanzausgleich in seine vertikale und horizontale Komponente. Alle Gemeinden erhalten (fiktiv) gleich hohe Pro-Kopf-Schlüsselzuweisungen $\left(Z_{\varnothing}\right)$. Die bestehenden Steuerkraftdifferenzen werden (ebenso fiktiv) durch Zuweisungen bzw. Abschöpfungen nivelliert. Die gewährten Schlüsselzuweisungen fassen beide Effekte gemäß Gleichung (7) zusammen. Für die kommunale Pro-Kopf-Finanzkraft (F) nach Finanzausgleich gilt nun:

(8) $\quad F=S+Z_{\varnothing}+a\left(S_{\varnothing}-S\right)$

Die Finanzkraft steigt im Durchschnitt an und alle Finanzkraftpositionen werden relativ zur durchschnittlichen Steuerkraft proportional herauf- bzw. herabgesetzt.

Der Grundbetrag variiert gemäß Gleichung (6) mit der Steuerkraft der Gemeinden, der Höhe der Schlüsselmasse und dem Ausgleichsatz:

- Der Grundbetrag steigt parallel zur durchschnittlichen Steuerkraft der Gemeinden: $\partial \mathrm{B}_{\varnothing}=\partial \mathrm{S}_{\varnothing}$. Der höhere Grundbetrag sorgt dafür, dass die auszugleichenden Finanzkraftdifferenzen im Durchschnitt konstant bleiben und die vorhandene Schlüsselmasse weiterhin vollständig ausgeschöpft wird.

- Darüber hinaus steigt der Grundbetrag, wenn das Land die Schlüsselmasse pro Kopf vergrößert: $\partial \mathrm{B}_{\varnothing}=\partial \mathrm{Z}_{\varnothing} / \mathrm{a}$. Davon profitieren alle Gemeinden in absolut gleichem Umfang: $\partial Z=\partial Z_{\varnothing}$.

- Schließlich vermindert ein Anstieg des Ausgleichsatzes den Grundbetrag so stark, dass die Durchschnittsgemeinde keine zusätzlichen Finanzzuweisungen erhält: $\partial \mathrm{B}_{\varnothing}=-\left(\mathrm{Z}_{\varnothing} / \mathrm{a}^{2}\right) \partial \mathrm{a}$. Der höhere Ausgleichsatz kommt den relativ steuerschwachen Gemeinden zugute, während die relativ steuerstarken Gemeinden Ausgleichsansprüche verlieren: $\partial \mathrm{Z}=\left(\mathrm{S}_{\varnothing}-\mathrm{S}\right) \partial \mathrm{a}$. 


\subsection{Funktion der Nivellierungshebesätze}

Bei der Ermittlung der Steuerkraftmesszahl geht es um die Erfassung des Steuerpotentials der Gebietskörperschaften. Soweit das Steueraufkommen beeinflussbar ist, darf im Finanzausgleich nicht das tatsächliche Aufkommen eingerechnet werden. Sonst würden die fiskalischen Konsequenzen autonomer Steuerpolitik die Höhe der Schlüsselzuweisungen verändern und könnten damit zum Teil auf andere Gemeinden abgewälzt werden. Beispielsweise würde ein unterdurchschnittlicher Hebesatzes bei der Gewerbesteuer zu höheren Finanzzuweisungen führen. Um diese Möglichkeit auszuschließen, werden zur Bestimmung der Steuerkraft im kommunalen Finanzausgleich einheitliche Nivellierungshebesätze verwendet.

Deren Bedeutung lässt sich anhand des Grundmodells unter der Annahme einer einzigen Steuer mit autonom bestimmbarem Hebesatz erläutern. Der kommunale Hebesatz (h) bezieht sich - wie bei der Gewerbesteuer und der Grundsteuer - auf einen Steuermessbetrag $(M)$, der die örtliche Steuerbemessungsgrundlage abbildet. Die Steuerkraft einer Gemeinde hängt von ihrem individuellen Steuermessbetrag ab, der jedoch mit dem für alle gleichen Nivellierungshebesatz $\left(h_{\varnothing}\right)$ multipliziert wird, um die Steuerkraft zu ermitteln. Für die Schlüsselzuweisungen gilt dann:

(9) $\quad Z=a\left(B_{\varnothing}-S\right)=a\left(B_{\varnothing}-h_{\varnothing} M\right)$

Offenkundig führt eine Veränderung des eigenen Hebesatzes nicht zu einer Veränderung der Steuerkraft und der Schlüsselzuweisungen.

Der Nivellierungshebesatz sollte dem landesdurchschnittlichen tatsächlichen Hebesatz entsprechen. Dies hat den Vorteil, dass die im Finanzausgleich insgesamt angerechnete Steuerkraft dem tatsächlichen Steueraufkommen entspricht. Eine durchschnittlich steuerstarke Gemeinde $\left(M=M_{\varnothing}\right)$ erhält weiterhin die mittlere Schlüsselzuweisung pro Kopf:

$$
Z_{\varnothing}=\mathrm{a}\left(\mathrm{B}_{\varnothing}-\mathrm{h}_{\varnothing} \mathrm{M}_{\varnothing}\right) \quad \Rightarrow \quad \mathrm{B}_{\varnothing}=\mathrm{h}_{\varnothing} \mathrm{M}_{\varnothing}+\mathrm{Z}_{\varnothing} / \mathrm{a}
$$

Der Grundbetrag, der das System zum Ausgleich bringt, hängt bekanntlich von der durchschnittlichen Steuerkraft ab. Diese wächst mit der Bemessungsgrundlage, aber auch mit dem Nivellierungshebesatz. Da ein steigender Nivellierungshebesatz nichts an der verfügbaren Schlüsselmasse ändert, muss er durch einen höheren Grundbetrag neutralisiert werden $\left(\partial \mathrm{B}_{\varnothing}=\mathrm{M}_{\varnothing} \partial \mathrm{h}_{\varnothing}\right)$. Per Saldo ändert sich nicht die Summe, aber die Verteilung der Schlüsselzuweisungen. Unter Berücksichtigung der Veränderung des Grundbetrags gilt für die Veränderung der Schlüsselzuweisungen: 
(11)

$$
\partial Z=a\left(\partial B_{\varnothing}-\partial h_{\varnothing} M\right)=a\left(M_{\varnothing}-M\right) \partial h_{\varnothing}
$$

Eine Anhebung des Nivellierungshebesatzes vergrößert die Schlüsselzuweisungen der relativ steuerschwachen Gemeinden $\left(M<M_{\varnothing}\right)$, während die steuerstarken Gemeinden weniger Schlüsselzuweisungen erhalten. Dies widerlegt einen verbreiteten Irrtum, denn die finanzschwachen Gemeinden befürchten stets, dass höhere Nivellierungshebesätze zu ihren Lasten gehen, weil ihnen eine höhere als die tatsächlich vorhandene Steuerkraft angerechnet wird. Sie übersehen dabei den aufgrund der Ausgleichsmechanik automatisch wachsenden Grundbetrag, der ihren Finanzbedarf vergrößert und allen unterdurchschnittlich steuerstarken Gemeinden zugute kommt.

Insgesamt zeigt sich, dass der kommunale Finanzausgleich keine echte Bedarfsorientierung aufweist. Es handelt sich vielmehr um ein System, das eine gegebene Finanzausgleichsmasse so auf die Kommunen verteilt, dass deren Finanzkraftdifferenzen verkleinert werden. Sollten diese Differenzen von sich aus schrumpfen, weil sich die Steuerkraft interkommunal gleichmäßiger verteilt, ändert sich das Finanzausgleichsvolumen nicht. Die Zuweisungen nähern sich dann einheitlichen Pro-Kopf-Beträgen und die vertikale Ausgleichskomponente tritt stärker in den Vordergrund.

\section{Typische Modifikationen des Grundmodells}

Im Folgenden wird gezeigt, wie sich Abweichungen vom reinen Finanzkraftausgleich, die in realen Finanzausgleichsystemen üblich sind, auf die Verteilung der Schlüsselzuweisungen auswirken.

\subsection{Abundante Gemeinden}

Der vertikale Ausgleich mit horizontalem Effekt kann im Prinzip wie ein horizontaler Direktausgleich unter den Gemeinden nach vorheriger vertikaler Aufstockung ihrer Finanzkraft funktionieren. Voraussetzung hierfür ist jedoch, dass alle Gemeinden mit ihrer vollständigen Steuerkraft in das System integriert werden. Dies wurde bislang implizit angenommen, denn Gleichung (7) für die Schlüsselzuweisungen enthält eine horizontale Umverteilungskomponente, die positive wie negative Werte annehmen kann: a $\left(S_{\varnothing}-S\right)$. Insgesamt verteilen sich die Schlüsselzuweisungen nach folgendem Muster:

- Eine durchschnittlich steuerstarke Gemeinde erhält die mittlere Schlüsselzuweisungen pro Einwohner. 
- Eine relativ steuerschwache Gemeinde erhält mehr, eine steuerstarke Gemeinde erhält weniger als die mittlere Pro-Kopf-Zuweisung.

Solange die gesamte Schlüsselzuweisung bei jeder Gemeinde einen positiven Wert hat, versteckt sich die Umverteilungskomponente in der gestaffelten Zahlung des Landes. Das System operiert dann technisch anders, aber mit gleicher Wirkung wie der rein horizontale Ausgleich. Es können aber so genannte abundante Gemeinden im Spiel sein, deren Steuerkraftmesszahl ihre Bedarfsmesszahl erreicht oder übersteigt: $\mathrm{S} \geq \mathrm{B}_{\varnothing}$. Sie erhalten nicht nur keine Schlüsselzuweisungen, sondern sind gemäß Gleichung (5) unter Umständen ausgleichspflichtig. Ihre über dem Bedarf liegende Finanzkraft wird zum Ausgleichsatz abgeschöpft und über die Schlüsselzuweisungen umverteilt.

Bei symmetrischer Gestaltung des vertikalen Finanzausgleichs sind die abundanten Gemeinden perfekt integriert und die Annäherung der Finanzkraftpositionen erfolgt gleichmäßig über das gesamte Finanzkraftspektrum. Wie bei einem horizontalen Direktausgleich profitieren steuerschwache Gemeinden von der Verteilung der Schlüsselzuweisungen, während die übrigen Gemeinden die Umverteilungslast in Form unterdurchschnittlicher Schlüsselzuweisungen bis hin zu Nettozahlungen tragen müssen.

Bei vertikalem Ausgleich mit horizontalem Effekt ist die Symmetrie jedoch meistens gestört, weil sich der Ausgleich nicht auf alle Gemeinden erstreckt, sondern nur auf Gemeinden, deren Bedarfsmesszahl über der Steuerkraftmesszahl liegt. Abundante Gemeinden erhalten zwar keine Schlüsselzuweisungen, müssen aber in der Regel auch nichts abgeben. Für die Schlüsselzuweisungen gilt dann:

Die Finanzkraft einer Gemeinde nach Schlüsselzuweisungen beläuft sich auf:

$$
\begin{aligned}
& \text { (13) } F=S+a\left(B_{\varnothing}-S\right) \quad \text { für } \quad B_{\varnothing}>S \\
& F=S \quad \text { für } \quad B_{\varnothing} \leq S
\end{aligned}
$$

Abundante Gemeinden stellen sich relativ zum horizontalen Direktausgleich besser, während die anderen Gemeinden über einen verhältnismäßig geringeren Grundbetrag belastet werden. Die Symmetrie der Nivellierungseffekte wird dadurch verletzt. Die Nichtabschöpfung der Abundanz trägt selbst zur Verschärfung des Problems bei, weil sich die Abundanzgrenze bei rückläufigem Grundbetrag nach unten verschiebt. 
Angesichts der asymmetrischen Ausgleicheffekte erscheint eine konsequente Integration der abundanten Gemeinden in den Finanzkraftausgleich wünschenswert. In der Praxis geschieht dies manchmal über eine spezielle Finanzausgleichsumlage, deren Abschöpfungssatz jedoch typischerweise unter dem allgemeinen Ausgleichsatz liegt. Damit wird faktisch ein gebrochen-linearer Umverteilungstarif verwendet, der das Abundanzproblem mildert, aber nicht beseitigt.

Für einen einheitlichen Ausgleichsatz auf positive wie negative Abweichungen zwischen Bedarfs- und Steuerkraftmesszahl sprechen Anreizaspekte. Die Grenzbelastungen, die bei wachsender eigener Steuerkraft durch den kommunalen Finanzausgleich entstehen, weil die Schlüsselzuweisungen abnehmen, wären für alle Gemeinden identisch. Ohne Abschöpfung der Abundanz ist die Grenzbelastung der abundanten Gemeinden dagegen gleich Null. Die Vergrößerung der Umverteilungsmasse könnte zudem für eine Verminderung des Ausgleichsatzes genutzt werden, was die Grenzbelastung aller anderen, vor allem der finanzschwachen Gemeinden reduzieren würde.

\subsection{Mindestfinanzkraftgarantie}

Reale Finanzausgleichsysteme beabsichtigen nicht nur eine proportionale Nivellierung der relativen Finanzkraftpositionen, sondern beinhalten in der Regel auch eine garantierte Mindestfinanzkraft. Besonders finanzschwache Gemeinden sollen dadurch in die Lage versetzt werden, ihre Aufgaben erfüllen zu können. Zu diesem Zweck werden die Schlüsselzuweisungen aufgestockt, wenn die Gemeinde mit allgemeinen Schlüsselzuweisungen einen Mindestprozentsatz $(\mathrm{m})$ ihrer Bedarfsmesszahl noch nicht erreicht:

(14) $\mathrm{F}=\mathrm{S}+\mathrm{a}\left(\mathrm{B}_{\varnothing}-\mathrm{S}\right)<\mathrm{m} \mathrm{B} \mathrm{B}_{\varnothing}$

Die Einhaltung der Mindestfinanzkraftgarantie erfordert ein bestimmtes Gesamtvolumen an Schlüsselzuweisungen:

$$
Z=\mathrm{mB}_{\varnothing}-\mathrm{S} \quad \text { für } \quad S<[(m-a) /(1-a)] B_{\varnothing}
$$

Die Mindestfinanzkraftgarantie reduziert das Volumen der Schlüsselzuweisungen, die zum Ausgleichsatz ausgeschüttet werden können. Der effektive Ausgleichseffekt muss dementsprechend kleiner ausfallen, was bei Konstanz des Ausgleichsatzes eine Reduktion der Bedarfsmesszahl bzw. des Grundbetrags impliziert $\left(\partial \mathrm{B}_{\varnothing}<0\right)$. Dies schmälert auch den realen Wert der Finanzkraftgarantie selbst, die sich auf die Bedarfsmesszahl und damit auf den flexiblen Grundbetrag bezieht $\left(\partial \mathrm{Z}=\mathrm{m} \partial \mathrm{B}_{\varnothing}\right)$. 
Eine Mindestfinanzkraftgarantie erzeugt Anreizprobleme, denn alle Gemeinden in der Garantiezone profitieren nicht von einer Erhöhung ihrer eigenen Steuerkraft, sondern verlieren in gleichem Umfang Finanzzuweisungen $(\partial Z=-\partial S)$. Bemühungen um eine Stärkung des eigenen Wirtschaftsstandortes zahlen sich fiskalisch nicht aus. Dieser klassische Konflikt zwischen Effizienz und Verteilung kann allenfalls entschärft, aber nicht beseitigt werden, z.B. durch einen progressiven Tarif, der finanzschwache Gemeinden hinreichend entlasten, aber die Abschöpfung der Steuerkraft unter 100\% senken würde.

\subsection{Bedarfsgewichtungen}

Die Annahme eines gleichen Pro-Kopf-Finanzbedarfs wird regelmäßig durch Einwohnergewichte im Hauptansatz oder Ergänzungsansätze durchbrochen. Diese erhöhen die individuelle Bedarfsmesszahl (B) nach Maßgabe eines relativen Bedarfsgewichts (g):

$$
B=g B_{\varnothing} \quad \text { mit } \quad g_{\varnothing}=1
$$

Der Einfachheit halber werden die Bedarfsgewichte im Durchschnitt auf 1 normiert. Der Effekt aller Bedarfsgewichte tritt so klar zutage: Bei gleicher Steuerkraftmesszahl erhalten Gemeinden mit $\mathrm{g}>1$ höhere und Gemeinden mit $\mathrm{g}<1$ geringere Pro-KopfZuweisungen als der Durchschnitt. Allerdings wird eine Erhöhung der Bedarfsmesszahl immer nur zum Teil kompensiert. Zudem fällt die Ausgleichsquote für die Gemeinden verschieden aus, wenn Mindestfinanzkraft und Abundanz traditionell geregelt sind. In diesem Fall existieren drei Tarifbereiche:

$$
\begin{array}{lll}
Z=m g B_{\varnothing}-S & \text { für } & S<g(m-a) /(1-a) B_{\varnothing} \\
Z=a\left(g B_{\varnothing}-S\right) & \text { für } & g[(m-a) /(1-a)] B_{\varnothing}<S<g B_{\varnothing} \\
Z=0 & \text { für } & g B_{\varnothing} \leq S
\end{array}
$$

Gemeinden, die mit dem normalen Ausgleichsatz die Mindestfinanzkraftgarantie nicht erreichen, erhalten eine Kompensation ihres Mehrbedarfs in Abhängigkeit von der Mindestgarantiequote $\left(\partial Z=\mathrm{m} \mathrm{B}_{\varnothing} \partial \mathrm{g}\right)$. Die übrigen nicht abundanten Gemeinden erhalten eine vom Normalsatz bestimmte Teilkompensation $\left(\partial \mathrm{Z}=\mathrm{a} \mathrm{B}_{\varnothing} \partial \mathrm{g}\right)$ und trotz Bedarfsgewichtung abundante Gemeinden gehen leer aus $(\partial Z=0)$.

Diese finanzkraftabhängige Dotierung von Sonderbedarfen ist nicht unproblematisch. Eine Gemeinde, die zentralörtliche Funktionen für das Umland ausübt, erhält zur Kompensation ihres Mehrbedarfs beispielsweise einen erhöhten Hauptansatz oder einen Ergänzungsansatz. Sie soll damit einer Gemeinde mit derselben Steuerkraft gleichgestellt 
werden, die den Sonderbedarf nicht aufweist. Solange Sonderbedarfe nur zum Teil ausgeglichen werden, gelingt dies jedoch nicht. Abundante Gemeinden mit Sonderbedarf erhalten im Vergleich zu anderen abundanten Gemeinden überhaupt keine Kompensation. Nachvollziehbare und ins Gewicht fallende Abweichungen vom Normbedarf sollten daher besser durch Zweckzuweisungen (mit einheitlicher Selbstbeteiligungsquote) außerhalb des Finanzkraftausgleichs dotiert werden (vgl. Scherf 2003, S. 22-23).

\subsection{Getrennte Schlüsselmassen}

In der Praxis ist eine Aufspaltung der Finanzausgleichsmasse in Teilschlüsselmassen für die kommunalen Gebietskörperschaftsgruppen üblich. Diese Vorgehensweise behindert eine gleichmäßige Nivellierung der Finanzkraftpositionen. Konsequent wäre es, kreisfreie Städte pro Einwohner genauso zu behandeln wie Landkreise und kreisangehörige Gemeinden zusammen, denn letztere erfüllen im kreisangehörigen Raum gemeinsam dieselben öffentlichen Aufgaben wie die kreisfreien Städte auf ihrem Gebiet.

In einem integrierten System werden Finanzkraft und -bedarf nach einheitlichen Kriterien ermittelt und ausgeglichen, während Systeme mit getrennten Schlüsselmassen das horizontale Ausgleichsziel zwischen und innerhalb der jeweiligen Gruppen, also zweistufig ansteuern müssen. Da in der Regel mit fixen Quoten an der Schlüsselmasse operiert wird, führt eine Verschiebung der Steuerkraft- oder der Bedarfsrelationen zwischen den Gruppen zwangsläufig zu unterschiedlichen Ausgleichsgraden, selbst wenn diese anfangs gut ausbalanciert waren. Teilschlüsselmassen erzeugen somit vermeidbare interkommunale Ungleichheiten.

Innerhalb der Landkreise kommt ein Problem hinzu. Dort erbringen die zentrale Kreisebene und die kreisangehörigen Gemeinden arbeitsteilig die öffentlichen Leistungen. Sie müssen über die Kreisumlage sicherstellen, dass beide Seiten entsprechend ihrer Aufgabenbelastung an den Gesamtkreiseinnahmen teilhaben. Deren originäre Verteilung wird jedoch vom Schlüsselzuweisungssystem mitbestimmt. Je mehr Schlüsselzuweisungen die Kreisgemeinden anstelle des Zentralkreises erhalten, um so höher ist der erforderliche Kreisumlagesatz.

Andererseits hängt der Finanzkraftausgleich unter den Gemeinden davon ab, dass die Schlüsselzuweisungen zunächst bei ihnen ankommen und ihre Finanzkraft horizontal differenziert erhöhen. Danach erfolgt die proportionale Abschöpfung der Gemeindefinanzkraft inklusive der erhaltenen Schlüsselzuweisungen durch die Kreisumlage. Sie 
lässt also die Finanzkraftrelationen zwischen den Gemeinden unberührt. Hier besteht ein gewisses Spannungsfeld zwischen dem horizontalen Ausgleichsziel, das eine Konzentration der Schlüsselzuweisungen auf die Gemeinden nahelegt, und dem dadurch forcierten, finanzpsychologisch ungünstigen Druck auf die Kreisumlage.

\section{Fazit}

Der kommunale Finanzausgleich in den Bundesländern ist vielgestaltig, komplex und intransparent. Die tatsächlichen Ausgleicheffekte verbergen sich hinter einem System kommunizierender Röhren, deren Verbindungen nicht deutlich werden. Generell neigen solche Systeme zu übertriebenem Perfektionismus. Ein höheres Einwohnergewicht für diese oder ein Sonderbedarf für jene Gruppe stimuliert ebenso berechtigt erscheinende Extraansprüche anderer Gemeinden. Dies spricht für eine Rückbesinnung auf das Grundmodell des begrenzten, alle Gemeinden gleichmäßig erfassenden Finanzkraftausgleichs und für den weitgehenden Verzicht auf die Berücksichtigung von Sonderbedarfen, die angesichts der insgesamt limitierten Finanzausgleichsmasse stets miteinander konkurrieren und sich teilweise kompensieren.

\section{Literatur}

Scherf, W. (2003), Sachgerechte Verteilung staatlicher Finanzzuweisungen, Finanzwissenschaftliche Arbeitspapiere 66-2003, Gießen.

Scherf, W. (2013), Kommunalfinanzen und Finanzausgleich, wisu 2/2013, S. 228-233.

Scherf, W. (2014), Steuerwettbewerb zwischen Effizienz und Gerechtigkeit, WiSt 11/2014, S. 608-613.

Kuhn, T. (1988), Schlüsselzuweisungen und fiskalische Ungleichheit, Frankfurt/Main.

Lenk, T., Hesse, M., Lück, O. (2013), Synoptische Darstellung der kommunalen Finanzausgleichssysteme der Länder aus finanzwissenschaftlicher Perspektive, Leipzig. 